
\title{
3 Research S Suare \\ Detection and Quantification of Bovine \\ Papillomavirus DNA by Digital Droplet PCR in Sheep Blood
}

\section{Sante Roperto ( $\square$ sante.roperto@unina.it )}

University of Naples Federico II

\section{Anna Cutarelli}

Istituto Zooprofilattico Sperimentale del Mezzogiorno

\section{Federica Corrado}

Istituto Zooprofilattico Sperimentale del Mezzogiorno

\section{Francesca De Falco}

University of Naples Federico II

\section{Canio Buonavoglia}

University of Bari Aldo Moro

\section{Research Article}

Keywords: bovine papillomavirus (BPV) DNA, digital droplet PCR (ddPCR), liquid biopsy, peripheral blood, viral load

Posted Date: January 28th, 2021

DOI: https://doi.org/10.21203/rs.3.rs-152338/v1

License: (c) (i) This work is licensed under a Creative Commons Attribution 4.0 International License. Read Full License 


\section{Abstract}

Highly pathogenic bovine Delta papillomaviruses (SPVs) were detected and quantified for the first time using digital droplet polymerase chain reaction (ddPCR) by liquid biopsy in 103 clinically healthy sheep. Overall, ddPCR detected bovine $\delta P V s$ in 68 blood samples (66\%). Bovine papillomavirus (BPV) infection by a single genotype was revealed in $59 \%$ of the blood samples, and BPV coinfection by double, triple or quadruple genotypes was observed in $41 \%$ of liquid biopsies. The BPV-2 genotype was most frequently seen in sheep, whereas BPV-1 was the least common. Furthermore, ddPCR was very useful for detection and quantification; the BPV-14 genotype was observed for the first time in ovine species, displaying the highest prevalence in some geographical areas (Apulia). In 42 of the positive samples (61.8\%), a single BPV infection was observed, 26 of which were caused by BPV-2 (61.9\%) and 7 by BPV-13 (16.7\%). BPV14 was responsible for 7 single infections (16.7\%) and BPV-1 for 2 single infections (4.7\%). Multiple BPV coinfections were observed in the remaining 26 positive samples (38.2\%), with dual BPV-2/BPV-13 infection being the most prevalent (84.6\%). BPV infection by triple and quadruple genotypes was also observed in $11.5 \%$ and $3.8 \%$ of cases, respectively. The present study showed that ddPCR, a biotechnological refinement of conventional PCR, is by far the most sensitive and accurate assay for BPV detection. Therefore, ddPCR displayed diagnostic and epidemiological value resulting in the identification of otherwise undetectable BPV genotypes as well as their geographical distributions and suggesting that animal husbandry practices contribute to cross-species transmission of BPVs..

\section{Introduction}

Papillomaviruses (PVs) comprise a diverse group of epitheliotropic, double-stranded DNA viruses that infect humans and animals in a species-specific manner [1]. PVs have co-evolved with their respective hosts, resulting in minimal cross-transfer between species [2]. Viruses such as PVs that slowly evolve with their hosts typically cause latent infection [3]. However, persistent infection by PVs causes a variety of severe diseases, including cutaneous and mucosal cellular proliferation [1].

Bovine papillomaviruses (BPVs) comprise 29 genotypes [4, 5]. Four highly pathogenic BPVs (BPV-1, -2, -13 , and -14) belong to the Delta genus ( $\delta P V s)$. They are associated with both cutaneous and mucosal benign and malignant tumors. Indeed, BPV-2 and BPV-13 are the most notable infectious agents commonly responsible for bladder tumors in some breeds of pasture-residing cattle that graze on lands rich in bracken fern (Pteridium spp.) [6, 7].

$\delta P V s$ are the only BPVs known to infect mesenchymal tissues and to show cross-species transmission and infection [1]. $\delta P V s$ have been detected in cutaneous wart lesions from ovines [8, 9]. Vertical transmission of $\delta P V s$ in sheep, resulting in oral fibropapillomatosis and epidermal hyperplasia of the lip in newborn lambs, has also been documented [10]. Furthermore, $\delta P V ~ D N A$ has been detected by polymerase chain reaction (PCR) in the peripheral blood of healthy sheep [11]. 
Although there are very limited numbers of reports describing BPV quantification data, PV studies have traditionally used real-time quantitative PCR (RT-qPCR) to measure the virus reservoir represented by PV DNA in both cutaneous and bladder neoplastic samples [12-15]. Most recently, digital PCR is gaining popularity as a novel approach to nucleic acid quantification as it allows for absolute target quantification. Indeed, digital droplet PCR (ddPCR) is a robust PCR technique that enables precise and accurate absolute quantification of target molecules by diluting and partitioning the samples into numerous compartments [16].

Quantification of PVs by digital PCR is proving to be a valuable improvement over qPCR, as it has been shown to have a higher robustness to mismatches between the primer-probe set and PV genotypes. Due to pathogens that cause latent infection, BPV concentrations in the blood are sometimes too low to be determined by traditional methods. In cattle and goats, ddPCR has been found to outperform RT-qPCR in terms of the sensitivity, specificity, and reproducibility of BPV detection, all of which play a central role in diagnostic and epidemiological procedures to identify the geolocalization of BPVs $[17,18]$.

The present study aimed to detect and quantify BPV DNA in the peripheral blood of sheep using ddPCR and to show the potential advantages of this molecular technology in the diagnosis and epidemiology of infectious diseases, including viral diseases.

\section{Materials And Methods}

\section{Liquid biopsy samples and DNA extraction}

Blood samples from 103 apparently healthy 1- to 3-year-old sheep were collected from the jugular vein in vacutainers containing ethylenediaminetetraacetic acid (EDTA). A total of 40 samples were obtained from sheep living in Sardinia (Sar) (20) and Campania (Cam) (20), 48 samples from Calabria (Cal) (24) and Basilicata (Bas) (24), and 15 samples from Apulia (Apu). All sheep, excluding those from Cam, were from flocks that lived and shared the bracken fern-infested lands that they grazed on with pastureresiding cattle. Sheep from Cam were from flocks living in closed pens without any contact with other animals. Total DNA was extracted using a DNeasy Blood \& Tissue Kit (Qiagen, Wilmington, DE, USA), according to the manufacturer's instructions.

\section{RT-qPCR}

RT-qPCR was performed in a final volume of $20 \mu \mathrm{L}$ containing $10 \mu \mathrm{L}$ of TaqMan Universal Master Mix (Applied Biosystems, Foster City, CA, USA), $900 \mathrm{nM}$ of each of the forward and reverse primers (Bio-Rad Laboratories, Hercules, CA, USA), $250 \mathrm{nM}$ of the probe (Bio-Rad Laboratories), and $100 \mathrm{ng}$ of the DNA sample. The primers and probes for the detection of four BPV genotypes (BPV-1, -2, -13, and -14) were used as reported elsewhere $[17,18]$. The reaction was performed on the CFX96 Real-Time System of the C1000 Touch $^{\mathrm{TM}}$ Thermal Cycler (Bio-Rad Laboratories). The thermal cycling conditions were as follows: $50^{\circ} \mathrm{C}$ for $2 \mathrm{~min}, 95^{\circ} \mathrm{C}$ for $10 \mathrm{~min}$, and 40 cycles of $95^{\circ} \mathrm{C}$ for $15 \mathrm{~s}$ and $58^{\circ} \mathrm{C}$ for $60 \mathrm{~s}$ (acquiring FAM and 
VIC dyes). Each sample was analyzed in duplicate, and negative controls were included in all runs. Data acquisition and data analyses were performed using CFX Maestro ${ }^{\mathrm{TM}}$ (Bio-Rad Laboratories) software. The same samples used as positive controls for ddPCR were also tested using RT-qPCR.

\section{DdPCR}

For ddPCR, Bio-Rad's QX100 ddPCR System was used according to the manufacturer's instructions. The reaction was performed in a final volume of $20 \mu \mathrm{L}$ containing $10 \mu \mathrm{L}$ of ddPCR Supermix for Probes (no dUTP 2x; Bio-Rad), $0.9 \mu \mathrm{M}$ primer, and $0.25 \mu \mathrm{M}$ probe together with $5 \mu \mathrm{L}$ sample DNA (100 ng). A black hole quencher was used in combination with FAM and VIC fluorescent dye reporters (Bio-Rad Laboratories). The reaction mixture was placed into the sample well of a DG8 cartridge (Bio-Rad Laboratories). A volume of $70 \mu \mathrm{L}$ of droplet generation oil was loaded into the oil well, and droplets were formed in the droplet generator (Bio-Rad Laboratories). After processing, the droplets were transferred to a 96-well PCR plate (Eppendorf, Hamburg, Germany). PCR amplification was carried out on a T100 Thermal Cycler (Bio-Rad Laboratories) with the following thermal profile: hold at $95^{\circ} \mathrm{C}$ for $10 \mathrm{~min}, 40$ cycles of $94^{\circ} \mathrm{C}$ for $30 \mathrm{~s}$ and $58^{\circ} \mathrm{C}$ for $1 \mathrm{~min}, 1$ cycle at $98^{\circ} \mathrm{C}$ for $10 \mathrm{~min}$, and ending at $4{ }^{\circ} \mathrm{C}$. After amplification, the plate was loaded onto a droplet reader (Bio-Rad Laboratories) and the droplets from each well of the plate were read automatically. QuantaSoft software was used to count the PCR-positive and PCR-negative droplets to provide absolute quantification of the target DNA. Therefore, the ddPCR results could be directly converted into copies/ $\mu \mathrm{L}$ in the initial samples simply by multiplying them by the total volume of the reaction mixture $(20 \mu \mathrm{L})$ and then dividing that number by the volume of DNA sample added to the reaction mixture $(5 \mu \mathrm{L})$ at the beginning of the assay. Samples with very few positive droplets were re-analyzed to ensure that these low copy number samples were not due to crosscontamination.

\section{Statistical analysis}

Differences in the proportions of detected cases were tested using the chi-square test by Campbell and Richardson [19]. Furthermore, regarding the significance relative to the number of copies of BPV DNA detected in sheep in the different regions, the t-test was used after adjusting for the Bonferroni multiple comparison correction of means. P-values $\leq .05$ were considered to be statistically significant. All analyses were performed using R statistical software (The R Foundation, Vienna, Austria).

\section{Results}

Overall, our results showed that BPV DNA was found in 68 out of 103 blood samples (66\%) from healthy sheep using ddPCR. The same liquid biopsies were also investigated using RT-qPCR, which revealed BPV DNA in approximately $9 \%$ of blood samples (Figure 1). In 42 of the positive samples (61.8\%), a single BPV infection was observed (Figure 2), 26 of which were caused by BPV-2 (61.9\%) and 7 by BPV-13 (16.7\%). BPV-14 was responsible for 7 single infections (16.7\%), and BPV-1 for two single infections 
(4.7\%) (Figure 3). Multiple BPV infections were seen in 26 (38.2\%) positive samples. BPV coinfections caused by two genotypes were seen in 22 positive cases (84.6\%), with dual BPV-2/BPV-13 infection being the most prevalent. BPV coinfections by triple and quadruple genotypes were detected in $11.5 \%(3 / 26)$ and $3.8 \%(1 / 26)$ of blood samples, respectively (Figure 4$)$. In sheep flocks that lived and shared lands with cattle, BPV DNA was detected in approximately $53 \%$ of blood samples collected in Apu (8/15), 75\% of samples acquired in both Bas and Cal (18/24), and $100 \%$ of blood samples harvested from Sar (20/20). In sheep flocks from Cam that lived in isolated and closed pens without any contact with cattle, BPV DNA was detected in $20 \%$ of blood samples examined (4/20). The percentage differences in BPV infections in all sheep flocks with cattle contact were statistically significant compared to the percentage observed in sheep flocks without any contact with cattle, as the Campbell-Ricardson's chi-square test resulted in a p-value $<0.05$. Furthermore, in all geographical areas except for Apu, BPV-2 was the most prevalent genotype. BPV-13 and BPV-14, as well as BPV-1 were also observed. Furthermore, Apu BPV-14 showed very high numbers of copies/ $\mu \mathrm{L}$ (mean value of 895.2); it was the most prevalent BPV genotype at a detection level of $40 \%$ in the examined samples (6/15) and a statistically significant CampbellRicardson's chi-square test $\mathrm{p}$-value $<.05$.

The overall quantification results showed that viral copy numbers/ $\mu \mathrm{L}$ ranged from 76 to 568 for BPV- 1 $($ mean value $=183.6), 65$ to 3768 for BPV-2 (mean value = 397.1), 66 to 2112 for BPV-13 (246.5), and76 to 1768 for BPV-14 (mean value $=447.5$ ). When sheep flocks from Sar alone were considered, BPV-2 showed the highest copy numbers/ $\mu \mathrm{L}$ with a mean of 1162 . Using t-tests, the differences between the copy numbers of BPV-2 in Sar compared to the other means found in Cal, Cam, Bas, and Apu were statistically significant with $\mathrm{p}$-values $<.05$. Indeed, after adjusting for the Bonferroni multiple comparison correction, their p-values were .003 (Sar-Cal), .04 (Sar-Cam), .002 (Sar-Bas), and .01 (Sar-Apu), respectively. Table 1 summarizes the quantitative data using QuantaSoft software, demonstrating the numbers of copies/ $\mu \mathrm{L}$ in blood samples from sheep flocks located in the five regions in South Italy. 
Table 1

Copy numbers/ $\mu \mathrm{L}$ of BPV DNA with the mean value and standard deviation (DS) of BPV DNA quantified by ddPCR in sheep blood samples.

\begin{tabular}{|lllll|}
\hline & BPV1 & BPV2 & BPV13 & BPV14 \\
Sar & 568,0 & 1162,0 & 254,5 & 387,9 \\
\hline Cal & 210,9 & 161,3 & 468,0 & 560,8 \\
\hline Cam & -- & 446,0 & 288,8 & 298,0 \\
\hline Bas & 139,2 & 121,3 & 136,3 & 95,8 \\
\hline Apu & -- & 95,0 & 85,0 & 895,2 \\
\hline & & & & \\
\hline MEAN & 183,6 & 397,1 & 246,5 & 447,5 \\
\hline SD & 233,4 & 450,1 & 149,3 & 301,2 \\
\hline
\end{tabular}

\section{Discussion}

To our knowledge, this study is the first to use the sensitive ddPCR assay, a biotechnological refinement of conventional PCR, to detect and quantify highly pathogenic bovine SPVs in clinically healthy sheep by liquid biopsy. Our previous studies have shown that ddPCR has both high specificity and sensitivity for the detection and quantification of BPV DNA in healthy and diseased cattle [17] as well as in healthy goats [18]. The current study provides further evidence that ddPCR is a very useful approach to detect and quantify BPV in the blood of healthy sheep and allows us to gain diagnostic and epidemiological insights into BPV presence in ovine species as data on the prevalence and types of BPVs in sheep are not currently available.

The liquid biopsy approach to the detection of circulating BPV DNA has garnered growing interest in PV studies [20]. Indeed, PV detection in the blood can be used as diagnostic, prognostic, and epidemiological markers [21].

Our study showed that BPV-2 is the most prevalent BPV genotype in healthy sheep, similar to other ruminants, such as cattle and goats. The highest number of copies of this genotype was found in Sar from sheep flocks in which cross-infections by BPVs have been previously reported [10], which suggests that copy numbers may correlate with the risk of cutaneous and mucosal lesions that progress to cancer. Furthermore, this study reports the first detection of BPV-14 in sheep. This genotype, chronologically the last BPV type identified in cattle, has never been described in the ovine species. Furthermore, our results demonstrated a statistically significant prevalence of BPV-14 in Apu compared to Sar, Cal, Bas, and Cam, which clearly showed that BPV genotype prevalence has a territorial divergence in these regions. 
We compared the sensitivity of ddPCR with that of RT-qPCR in evaluating the same liquid biopsy, demonstrating that ddPCR has superior sensitivity compared to RT-qPCR. Therefore, our results suggest that ddPCR is by far the most sensitive and accurate assay for BPV detection. It is worth noting that it has been shown that ddPCR outperforms RT-qPCR in terms of the sensitivity, specificity, and reproducibility of oncogenic human papillomavirus detection and quantification [22-24].

BPV genotype detection in the blood of healthy sheep suggests that the bloodstream can be the primary site of BPV infection. As bovine $\delta P V s$ are known to infect ovine species and result in anatomoclinical diseases, it is conceivable that these viruses may spread through the blood, which could be responsible for secondary tissue localization and infection. However, as in humans [1], further epidemiological studies are required to enhance the understanding of BPV transmission via the bloodstream.

Here, we detected a higher percentage of BPVs in sheep that were in close contact with cattle herds, about which numerous case reports of BPV infection have been described. Indeed, the sheep flocks in our study shared bracken fern-infested lands with pasture-residing cattle for grazing. The evidence from epidemiological studies of cattle is sufficiently strong to suggest that in the presence of BPV infection, the toxic components of bracken ferns such as ptaquiloside (PT), a water-soluble norsesquiterpenoid glycoside, are ecological co-factors in the development of severe diseases due to BPVs, including chronic enzootic hematuria (CEH), a clinical syndrome caused by bladder tumors [25]. Thus, it is conceivable that PT may also be a co-factor of diseases in sheep. Indeed, PT has recently been detected in biological matrices from healthy sheep [26]. PT is known to hamper the immune system and may play an important role in cross-species transmission and infection of bovine SPVs. It is worth noting that outbreaks of $\mathrm{CEH}$ have also been reported in sheep $[27,28]$. Furthermore, bovine $\delta P V$ infection resulting in clinical disease are known to occur in sheep [8-10]. Therefore, the detection of bovine $\delta P V$ DNA in the blood of sheep means that sheep can be infected by these PVs, which may make $\delta P V s$ an additional, potential cause of ovine disease. Furthermore, our results suggest that clinically healthy sheep may represent a reservoir for bovine $\delta P V s$. Thus, it is conceivable that sheep may play a role in intra- and interspecies bovine $\delta P V$ transmission and infection. In this context, very precise quantitation of very low viral copy numbers can provide more precise monitoring of latent BPV DNA reservoirs.

Finally, it is well known that PV distribution varies considerably by geography [29]. Therefore, ddPCR may be an essential tool for improving diagnostic procedures thus allowing the identification of the genotypic distribution of BPV and a better understanding of the possible, geographical divergence of BPV prevalence in different areas. The ddPCR assay appears to possess high sensitivity and accuracy, which is valuable for addressing the molecular burden of BPV infections and useful for defining an accurate ecological epidemiology. This baseline information improves our knowledge about the molecular mechanisms of the disease and provides insights into necessary measures for reducing the risk of BPV infection and/or co-infection.

In conclusion, ddPCR is presently being used to detect very low nucleic acid concentrations and, therefore, appears to be of interest in the diagnosis of infectious diseases, including viral diseases [30]. DdPCR has 
proven to be a valuable new technology and with additional improvements in prospect it is likely to become an indispensable tool in diagnostic, prognostic and epidemiological virus research [31]. Therefore, the ddPCR method may provide a new and promising tool for evaluating the BPV viral load in clinical samples. Future PV research warrants the use of this molecular approach to assess PV typespecific pathogenetic pathways of disease, including carcinogenicity. Indeed, available evidence from BPV distribution lends strong support to the notion that the risk of an animal developing a BPVassociated disease varies substantially according to the specific BPV type with which the animal is infected. Finally, the ddPCR approach may provide a better understanding of the complex interactions between multiple BPV types during coinfections, as the possible interference resulting from multiple PV genotypes in coinfection cases remains an open question [32].

\section{Abbreviations}

Apu: Apulia

Bas: Basilicata

BPV: bovine papillomavirus

Cal: Calabria

Cam: Campania

CEH: chronic enzootic hematuria

ddPCR: digital droplet polymerase chain reaction

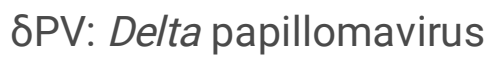

dUTP: 2'-Deoxyuridine, 5'-Triphosphate

EDTA: ethylenediaminetetraacetic acid

FAM: Fluorescein amidite

PCR: polymerase chain reaction

PT: ptaquiloside

PV: papillomavirus

RT-qPCR: real time quantitative polymerase chain reaction

Sar: Sardinia

VIC: Victoria 


\section{Declarations}

\section{Ethics statement}

Blood samples were collected from animals in public slaughterhouses during the mandatory antemortem clinical examination. All procedures performed in this study followed common good clinical practices and received institutional approval from the Ethical Animal Care and Use Committee of the University of Naples Federico II (PG/2017/ 0099607). All farmers were previously informed and in agreement with the purpose and methods used.

\section{Consent for publication}

Not applicable

\section{Availability of data and materials}

All data generated and/or analyzed during the current study are available from the corresponding author on reasonable request

\section{Declaration of conflicting interests}

The authors declare that they have no competing interests.

\section{Funding}

This research was partly supported by grants from the Regione Campania and Regione Basilicata. The funders of the work did not influence the study design, data collection and analysis, decision to publish, or preparation of the manuscript.

\section{Acknowledgements}

We would like to thank Dr. A. Venuti, IRCSS Regina Elena National Cancer Institute, (Rome, Italy) for providing BPV-2-positive samples as a kind gift as well as Dr. G. Salvatore, of Regione Basilicata, Drs. E. Grieco and S. Milone from Azienda Sanitaria Locale (ASL) of Salerno, Dr R.N. La Rizza from ASL of Vibo Valentia, Dr. Domenico Rufrano, scholarship holder of Naples University, and Dr. S. Morace, University of Catanzaro “Magna Graecia” for their technical assistance.

\section{Authors' contributions}


SR designed this study; AC, FDF performed the experiments; CF, SR analyzed the data; SR and CB drafted and revised the manuscript. All authors read and approved the final manuscript.

\section{References}

1. IARC Monographs on the Evaluation of Carcinogenesis risk to Humans. (2007). Vol. 90: Human Papillomavirus, World Health Organization, Ed., Lyon, France: WHO Press, p 47.

2. Doorbar J, Egawa N, Griffin H, Kranjec C, Murakami I (2016). Human papillomavirus molecular biology and disease association. Rev Med Virol 25, (Suppl. 1) 2-23.

3. Antonsson A, McMillan NAJ (2006). Papillomavirus in healthy skin of Australian mammals. J Gen Virol 87, 3195-3200.

4. http://pave.niaid.nih.gov/; Accessed 15 January 2021

5. Yamashita-Kawanishi N, Ito S, Ishiyama D, Chambers JK, Uchida K, Kasuya F, Haga, T (2020). Characterization of bovine papillomavirus 28 (BPV28) and a novel genotype BPV29 associated with vulval papillomas in cattle. Vet Microbiol 250, 108879, doi 10.1016/j.vetmic.2020.108879.

6. Campo MS, Jarrett WF, Barron R, O'Neil BW, Smith KT (1992). Association of bovine papillomavirus type 2 and bracken fern with bladder cancer in cattle. Cancer Res 52, 6898-6904.

7. Borzacchiello G, lovane G, Marcante ML, Poggiali F, Roperto F, Roperto S, Venuti A (2003). Presence of bovine papillomavirus type 2 DNA and expression of the viral oncoprotein E5 in naturally occurring urinary bladder tumours in cows. J Gen Virol 84, 2921-2926.

8. Mazzucchelli-de-Souza J, de Carvalho RF, Módolo DG, Thompson CE, Araldi RP, Stocco R.C. (2018). First detection of bovine papillomavirus type 2 in cutaneous wart lesions from ovine. Transbound Emerg Dis 65, 939-943.

9. Savini F, Gallina L, Prosperi A, Puleio R, Lavazza A, Di Marco P, Tumino S, Moreno A, Lelli D, Guercio A, Scagliarini A (2020). Bovine papillomavirus 1 gets out of the flock: detection in an ovine wart in Sicily. Pathogens 9, 429, doi: 10.3390/pathogens9060429.

10. Roperto S, Russo V, Corrado F, De Falco F, Munday JS, Roperto F (2018a). Oral fibropapillomatosis and epidermal hyperplasia of the lip in newborn lambs associated with bovine Deltapapillomavirus. Sci Rep 8(1), 13310, doi: 10.1038/s41598-018-31529-9.

11. Roperto S, Russo V, Corrado F, Munday JS, De Falco F, Roperto F (2018b). Detection of bovine Deltapapillomavirus in peripheral blood of healthy sheep (Ovis aries). Transbound Emerg Dis 65, 758-764.

12. Yuan Z, Gallagher A, Gault EA, Campo MS, Nasir L (2007). Bovine papillomavirus infection in equine sarcoids and in bovine bladder cancers. Vet J 174, 599-604.

13. Pangty K, Singh S, Goswami R, Saikumar G, Somvanshi R (2010). Detection of BPV-1 and -2 and quantification of BPV-1 by real-time PCR in cutaneous warts in cattle and buffaloes. Transbound Emerg Dis 57, 185-196. 
14. Pathania S, Dhama K, Saikumar G, Shahi S, Somvanshi R (2012). Detection and quantification of bovine papilloma virus type 2 (BPV-2) by real-time PCR in urine and urinary bladder lesions in enzootic bovine haematuria (EBH)-affected cows. Transbound Emerg Dis 59, 79-84.

15. Cota JB, Peleteiro MC, Petti L, Tavares L, Duarte A (2015). Detection and quantification of bovine papillomavirus type 2 in urinary bladders and lymph nodes in cases of Bovine Enzootic Hematuria from the endemic region of Azores. Vet Microbiol 178, 138-143.

16. Kanagal-Shamanna R (2016). Digital PCR: Principles and Applications. Methods Mol Biol 1392, 4350.

17. De Falco F, Corrado F, Cutarelli A, Leonardi L, Roperto S (2020). Droplet digital PCR for the detection and quantification of circulating bovine Transbound Emerg Dis, doi:10.1111/tbed.13795.

18. Cutarelli A, De Falco F, Uleri V, Buonavoglia C, Roperto S (2021). The diagnostic value of the droplet digital PCR for the detection of bovine Deltapapillomavirus in goats by liquid biopsy. Transbound Emerg Dis, doi:1111/tbed.13971.

19. Richardson J T E (2011). The analysis of 2 X 2 contingency tables - - Yet again. Stat Med 30, 890, doi: $10.1002 / \operatorname{sim} .411$.

20. Veyer D, Wack M, Mandavit M, Garrigou S, Hans S, Bonfils, P., Tartour E, Bélec L, Wang-Renault SF, Laurent-Puig P, Mirghani H, Rance B, Taly V, Badoual C, Péré H (2020). HPV circulating tumoral DNA quantification by droplet-based digital PCR: a promising predictive and prognostic biomarker for HPV-associated oropharyngeal cancers. Int J Cancer 147, 1222-1227.

21. Bishop JA, Ha PK (2016). Human papillomavirus detection in a "Digital" age. Cancer 122, 1502-1504.

22. Biron VL, Kostiuk M, Isaac A, Puttagunta L, O'Connell DA, Harris J, Côté DW, Seikaly H (2016).

Detection of human papillomavirus type 16 in oropharyngeal squamous cell carcinoma using droplet digital polymerase chain reaction. Cancer 122, 1544-1551.

23. Jeannot E, Becette V, Campitelli M, Calméjane MA, Lappartient E, Ruff E, Saada S, Holmes A, Bellet D, Sastre-Garau X (2016). Circulating human papillomavirus DNA detected using droplet digital PCR in the serum of patients diagnosed with early stage human papillomavirus-associated invasive carcinoma. J Pathol Clin Res 2, 201-209.

24. Carow K, Read C, Häfner N, Runnebaum IB, Corner A, Dürst M (2017). A comparative study of digital PCR and real-time qPCR for the detection and quantification of HPV mRNA in sentinel lymph nodes of cervical cancer patients. BMC Res Notes 10, 532, doi: 10.1186/s13104-017-2846-8.

25. Smith BL (1990). Bracken fern and animal health in Australia and New Zealand. In: Thompson, J.A., Smith, B.L. (Eds), Bracken Biology and Management, Australian Institute of Agricultural Science, Occasional Publication No. 40, 227-232.

26. Virgilio A, Sinisi A, Russo V, Salvatore G, Santoro A, Galeone A, Taglialatela-Scafati O, Roperto F (2015). Ptaquiloside, the major carcinogen of bracken fern, in the pooled raw milk of healthy sheep and goats: an underestimated, global concern of food safety. J Agric Food Chem 63, 4886-4892. 
27. Prakash AS, Pereira TN, Smith BL, Shaw G, Seawright AA (1996). Mechanism of bracken fern carcinogenesis: evidence for $\mathrm{H}$-ras activation via initial adenine alkylation by ptaquiloside. Nat Toxins 4, 221-227.

28. Cianciolo RE, Mohr FC (2016). Urinary system. In: Maxie, M.G. (Ed.), Jubb, Kennedy and Palmer's Pathology of Domestic Animals, $6^{\text {th }}$ Edition, vol 2, Elsevier, St Louis, Missouri, pp 376-474.

29. Chen Z, Li Q, Huang Q, Liu H, Jiang H, Chen Z, An Z, Luo Q (2019). Characteristics of human papillomaviruses distribution in Guizhou Province, China. Virol J 16, 123, doi: 10.1186/s12985-0191239-0.

30. Li H, Bai R, Zhao Z, Tao L, Ma M, Ji Z, Jian M, Ding Z, Dai X, Bao F, Liu A (2018). Application of droplet digital PCR to detect the pathogens of infectious diseases. Biosci Rep 38, BSR20181170. doi: 10.1042/BSR20181170.

31. Rutsaert S, Bosman K, Trypsteen W, Nijhuis M, Vandekerckhove L (2018). Digital PCR as a tool to measure HIV persistence. Retrovirology 25, 16, doi: 10.1186/s12977-018-0399-0.

32. Biryukov J, Meyers C. (2018). Superinfection exclusion between two high-risk human papillomavirus types during coinfection. J Virol 92, e01993-17, doi: 10.1128/JVI.01993-17.

\section{Figures}

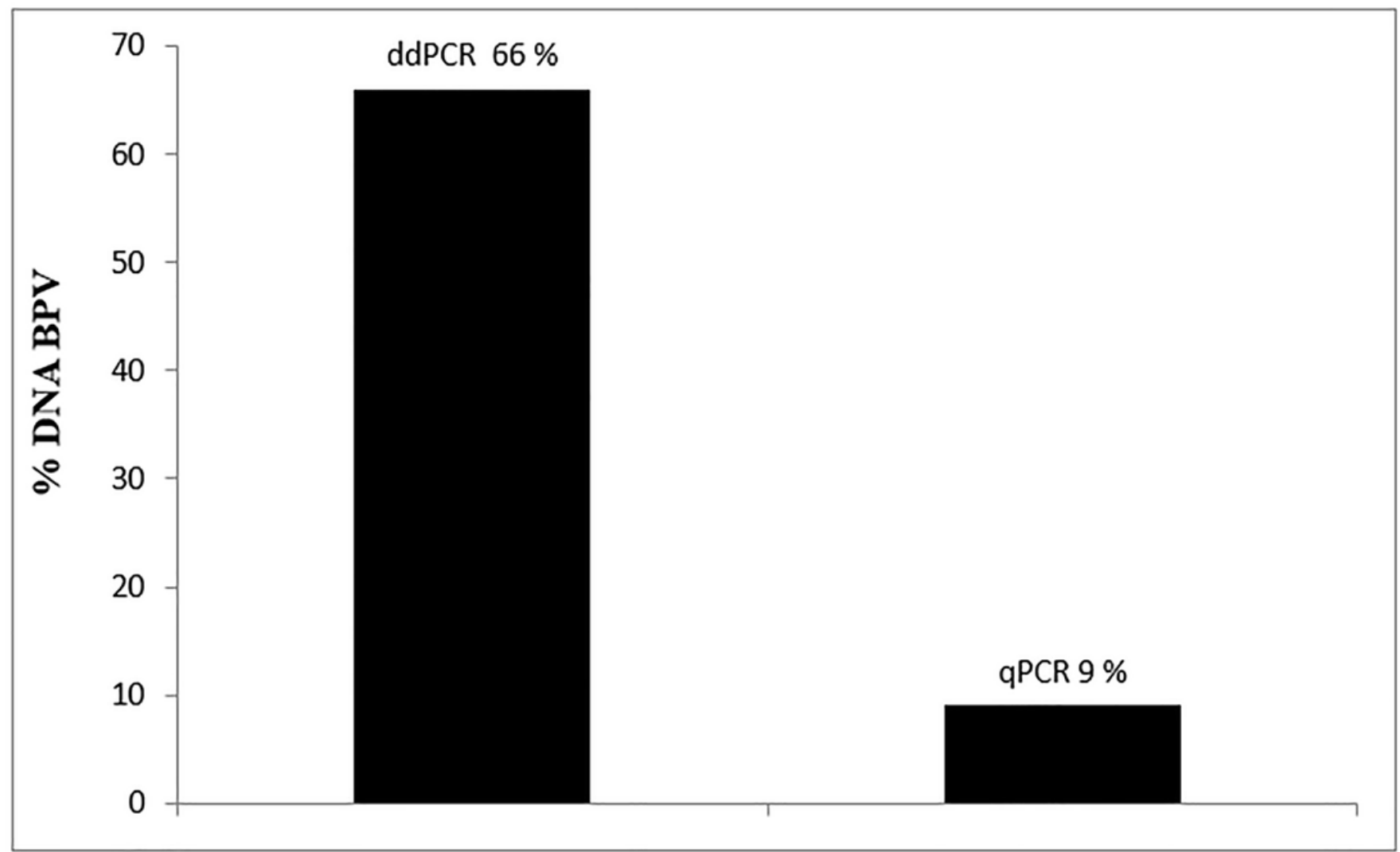


Figure 1

Percentage of circulating BPVs detected by ddPCR and RT-qPCR in blood samples from healthy sheep.

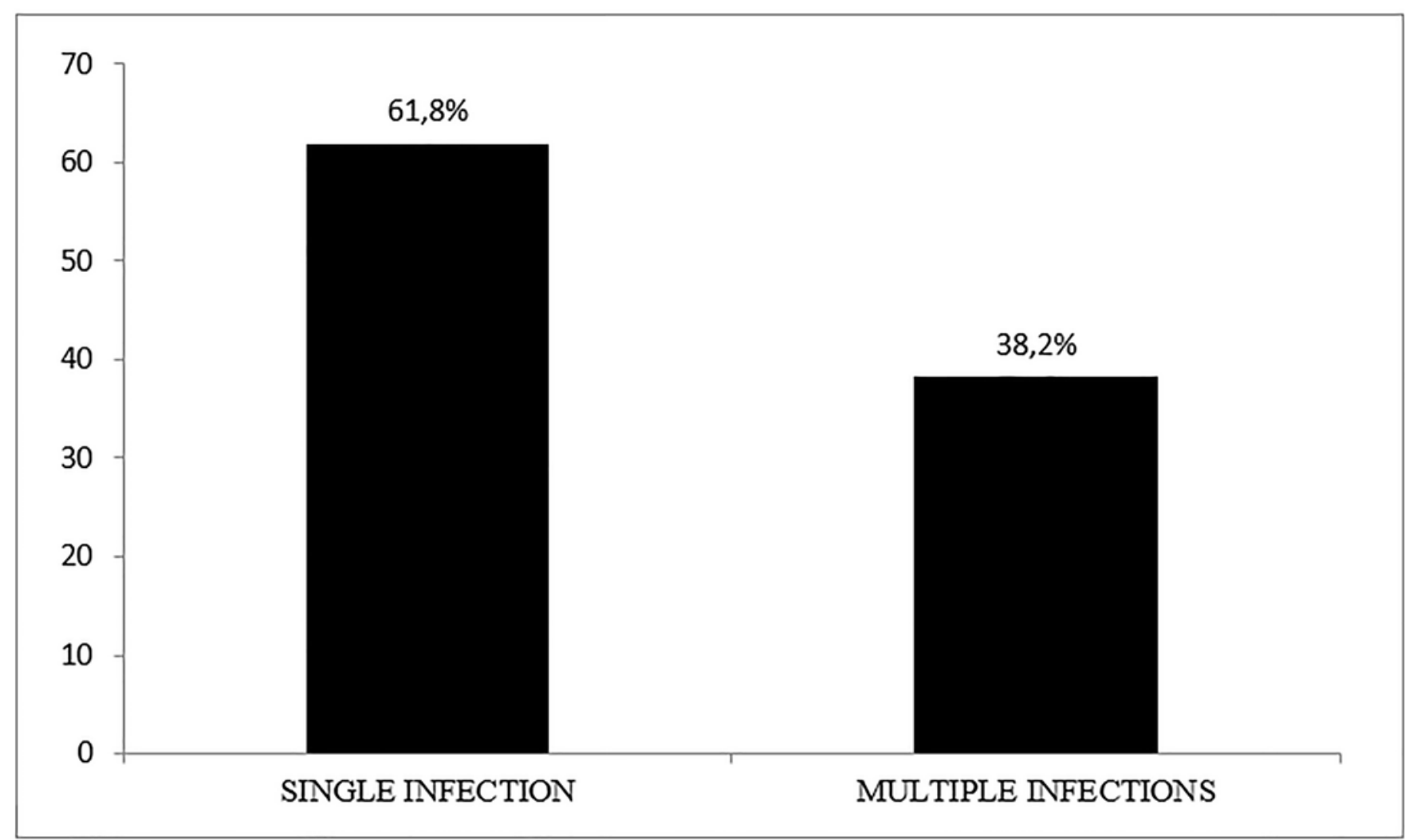

Figure 2

Detection of circulating BPVs. Single and multiple BPV genotypes were detected. 


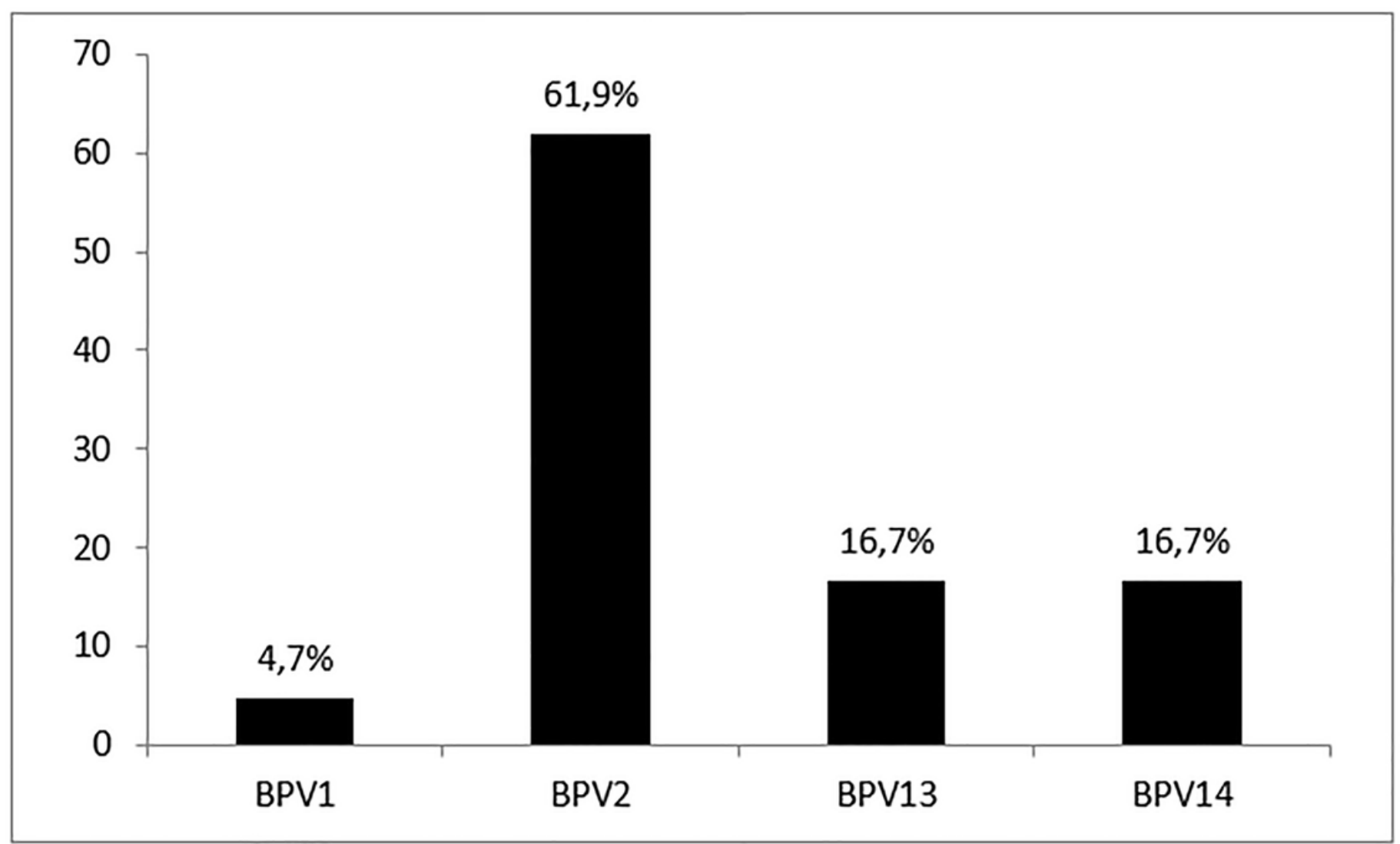

Figure 3

Overall percentage of circulating BPVs by single genotypes. BPV2 was by far the most prevalent genotype detected in all geographical regions. 


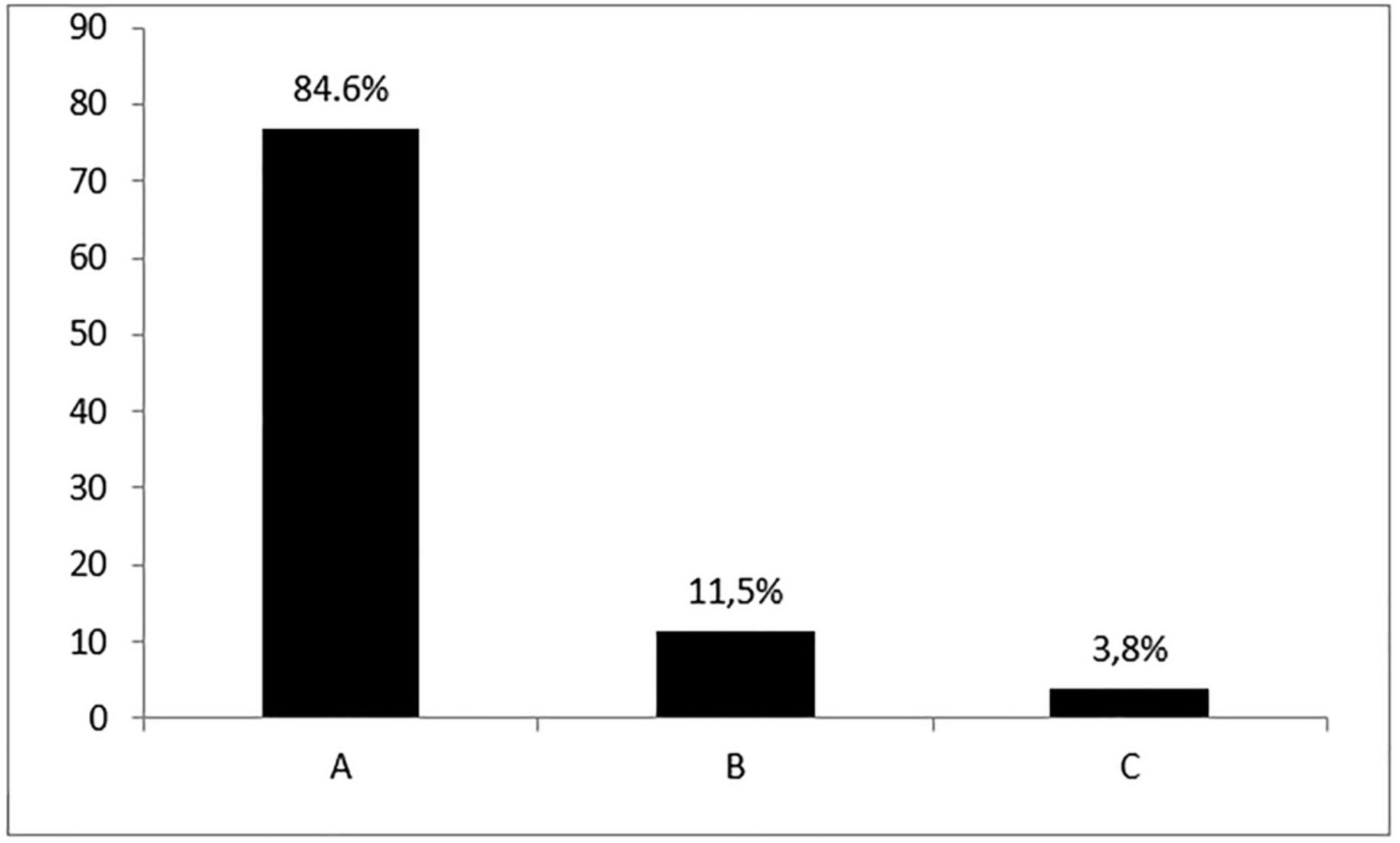

Figure 4

BPV coinfection by double genotypes (A) represented the majority of virus infections by multiple types. Coinfections by triple (B) and quadruple (C) genotypes were rarely seen. 